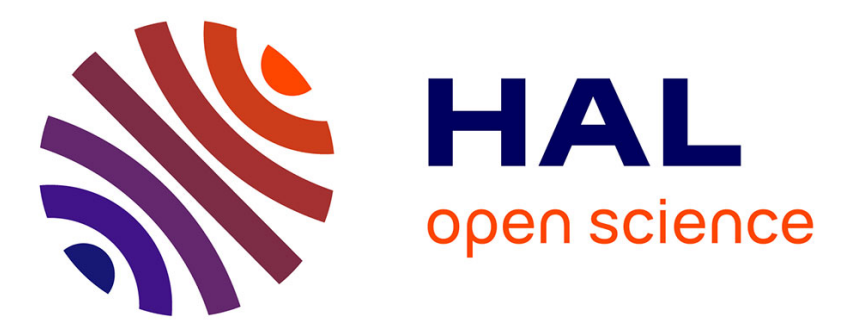

\title{
Optimization of multi-period supply planning under stochastic lead times and a dynamic demand
}

Oussama Ben-Ammar, Belgacem Bettayeb, Alexandre Dolgui

\section{To cite this version:}

Oussama Ben-Ammar, Belgacem Bettayeb, Alexandre Dolgui. Optimization of multi-period supply planning under stochastic lead times and a dynamic demand. International Journal of Production Economics, 2019, 218, pp.106-117. 10.1016/j.ijpe.2019.05.003 . hal-02435962

\section{HAL Id: hal-02435962 \\ https://hal.science/hal-02435962}

Submitted on 21 Jan 2020

HAL is a multi-disciplinary open access archive for the deposit and dissemination of scientific research documents, whether they are published or not. The documents may come from teaching and research institutions in France or abroad, or from public or private research centers.
L'archive ouverte pluridisciplinaire HAL, est destinée au dépôt et à la diffusion de documents scientifiques de niveau recherche, publiés ou non, émanant des établissements d'enseignement et de recherche français ou étrangers, des laboratoires publics ou privés. 


\title{
Optimization of multi-period production planning under stochastic lead times and a dynamic demand
}

\author{
Oussama Ben-Ammar ${ }^{1}$, Belgacem Bettayeb ${ }^{2}$, Alexandre Dolgui ${ }^{1}$ \\ IMT Atlantique, LS2N, UMR-CNRS 6004, La Chantrerie, 4 rue Alfred Kastler, 44300 Nantes, France \\ CESI Group, LINEACT Lab, 1 Rue G. Marconi, Mont-Saint-Aignan, France
}

\{oussama.ben-ammar, alexandre.dolgui\}@imt-atlatique.fr, bbettayeb@cesi.fr

\begin{abstract}
Production planning and inventory control in supply chain are of prime importance for companies which aim to produce high quality Finished products at lowest costs and right on time. For this reason, planners must reduce average stock levels and determine optimal safety lead times. This study deals with a multi-period production planning problem with a known dynamic demand. The lead times of demands are independent, discrete random variables with known and bounded probability distributions. A general probabilistic model, including a recursive procedure to calculate the expected total cost, is derived. A Genetic Algorithm is developed for this model to determine planned lead times and safety stock level which minimize the total expected cost. The latter is equal to the sum of the backlogging and inventory holding costs. This approach is compared to three other ones to illustrate its performance. The results prove that, under certain assumptions, it could be advantageous to optimizing planned lead times rather than implementing safety stocks. To understand the effect of dispersion on the robustness of the solution, different levels of variance and different shapes of lead time distributions are studied. Different analysis proves that the variability of the lead time affects slightly the expected total cost when the unit inventory holding cost is close to the unit backlogging cost.
\end{abstract}

Keywords: Production planning, Multi-period, Dynamic demand, Stochastic lead times, Genetic Algorithm.

\section{Introduction and related publications}

Managing uncertainty is becoming one of the most important challenges in supply chain optimization. In fact, uncertainty causes several difficulties in production planning and inventory control. The sources of uncertainty are various and can take place at several levels of the supply chain: demand variability, machines breakdowns, transport delays, quality issues, etc. In this paper, we investigate the case of single stage production planning with a lot for lot policy where demands are known and should be delivered at given due dates. Once a demand is released, the delivery takes place after a random lead time. This randomness causes backlogging and inventory holding costs. The objective is to minimize the total expected cost by optimizing the planned lead times and the safety stock.

Without attempting exhaustive review of the literature, we will point out and discuss some important studies relevant to the present research. To better anticipate uncertainty of lead times, planners use several techniques such as safety lead times and safety stocks. Jansen and de Kok (2013) highlighted the importance of anticipating lead times while Chopra et al (2004), He (2005) and Van Kampen et al. (2010) explained in which cases it could be advantageous to consider safety lead times and/or safety stocks.

Among the first models considering one-stage production system deterministic demand and stochastic processing time, Weeks (1981) has proven that the problem is equivalent to the standard Newsboy problem when lead times are independent and identically distributed. Using the latter assumption, several other approaches have been proposed to determine optimal ordering policies (Porteus, 2002, Muthuraman et al. 2014). For other models considering stochastic lead times, but in the case of multiple suppliers, readers can refer to the review of Minner (2003). In the case of assembly systems, the literature review can be split into two different cases: (i) single period planning (Yano 1987, Chu et al. 1993, Tang and Grubbström, 2003, Axsäter 2005, and Ben-Ammar et al. 2017); and (ii) multi-period planning (see for 
example the paper Ould Louly and Dolgui 2013 and references included) where the demand is usually considered as constant. Readers can refer to Dolgui et al. (2013) for more exhaustive review.

Close to our model, Deng et al. (2010) investigated a single stage inventory model with both stochastic demand and lead times. The goal is to determine an optimized sequence of orders, while minimizing the expected total cost over a discrete finite horizon. By assuming that the distribution of lead times degenerates to zero, a simulated annealing algorithm is introduced to solve the problem. Similarly, Rossi et al. (2012) addressed the same problem and developed fast heuristic approaches.

In this paper, we study the case where lead times are independent discrete random variables with known and bounded probability distributions, but not necessarily identically distributed. Demands are supposed deterministic but not constant. To the best of our knowledge, no such study has been reported in the literature.

The rest of the paper is organized as follows. Section 2 gives the problem formulation and considered assumptions. The proposed resolving approaches are detailed in Section 3. Some results are presented and discussed in Section 4. Finally, Section 5 is devoted to the conclusions and the perspectives.

\section{Problem formulation}

The details of the stochastic multi-period production planning are as follows. A sequence of dynamic demands, denoted by $D_{t}$, for a single product is placed over a finite planning horizon of $T$ discrete periods and can be non-null between periods $p_{m}$ and $p_{M}$ (see Figure 1). Each demand $D_{t}$ is launched at period $t-X_{t}$ where $X_{t}$ is its planned lead time, whereas $L_{t}$, its real lead time, is a discrete random variable varying between $L_{t}^{-}$and $L_{t}^{+}$. The lead times of demands are independent but not necessarily identically distributed. At the beginning of each period $t$ we may receive the quantity $A_{t}$ which is the sum of demands $D_{j}$ ordered at periods $j-X_{j}$ such as $j-X_{j}+L_{j}=t$. The set of indexes of demands verifying the last condition is denoted by $\mathcal{A}_{t}$. Note that we may receive a non-null quantity between $p_{h}$, the first period with a possible nonnull inventory level, and $p_{B}+1$, where $p_{B}$ the index of the last period with a possible non-null backlog quantity (see Figure 1).

Each demand can be supplied only from on-hand inventory if it is positive. If the inventory is null, the unsatisfied demands will increase the backlog level of the period and will stay in the system until they are satisfied. Note that backlogging may occur on periods $p_{b}, \ldots, p_{B}$ where $p_{b}=p_{m}$ because we cannot have backlogging until the first period with non-null demand (see Figure 1). To calculate the backlog quantity at each period $t \in\left\{p_{b}, \ldots, p_{B}\right\}$, we use the set $\mathcal{M}_{t}$ which contains the indexes of demands $D_{j}$ that are involved.

One of the following costs may be non-null at the end of each period $t$ : (i) if the inventory level is positive, the incurred cost is evaluated using unit inventory holding cost denoted by $c^{h}$, (ii) if there is a shortage, the incurred cost is proportional to the unit backlogging cost $c^{b}$. To tackle the effects of uncertainty in inventory and production planning, a safety stock, denoted by $S_{0}$ is introduced as a decision variable.

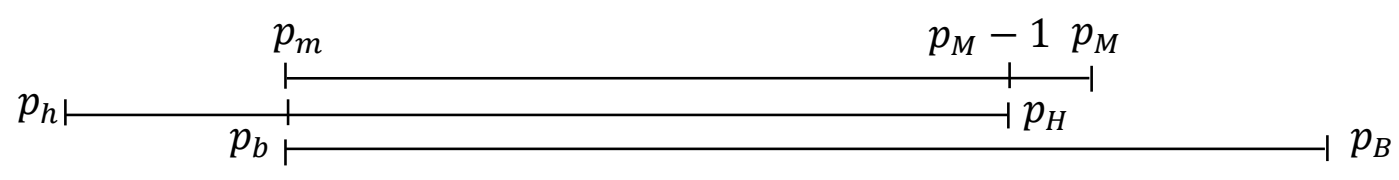

Figure 1. Ranges of periods of demands, no null inventory and no null backlogging.

\section{$2.1 \quad$ Notations}

The full list of notations is given below: 


\section{Parameters and Variables}

$t \quad$ Index of the period

$D_{t} \quad$ Demand at period $t$

$p_{m} \quad$ Index of the first period with a non-null demand

$p_{M} \quad$ Index of the last period with a non-null demand

$X_{t} \quad$ Planned lead time of period $t$, it is a decision variable

$L_{t} \quad$ Real lead time of the demand at period $t$ which is launched at period $t-X_{t}$, it is a discrete random variable varying between $L_{t}^{-}$and $L_{t}^{+}$

$A_{t} \quad$ Release order quantity at period $t$

$\mathcal{A}_{t} \quad$ Set of indexes of demands $D_{j}$ ordered at periods $j-X_{j}$ that may be received at period $t$. A period $j \in \mathcal{A}_{t}$ implies that $j \in\left\{p_{m}, \ldots, p_{M}\right\}$ and $L_{j}^{-} \leq t-j+X_{j} \leq L_{j}^{+}$

$\mathcal{M}_{t} \quad$ Set of indexes of periods involved in the calculation of the expected value of the backlog quantity at period $t$. A period $j \in \mathcal{M}_{t}$ implies that $j \in\left\{p_{m}, \ldots, p_{M}\right\}$ and $L_{j}^{-} \leq t-j+$ $X_{j} \leq L_{j}^{+}-1$

$m_{t} \quad$ First element in the set $\mathcal{M}_{t}$

$M_{t} \quad$ Last element in the set $\mathcal{M}_{t}$

$R_{t} \quad$ Backlogging value at period $t$

$S_{t} \quad$ Inventory holding value at period $t$

$\mathcal{R}_{t}^{\alpha} \quad$ Subset of sample space of $R_{t}$ where only $\alpha$ demands arrive before or at period $t$

$r_{t}^{\alpha} \quad$ Realization of random variable $R_{t}$ where $\alpha$ is the number of demands $D_{j}, j \in \mathcal{M}_{t}$ arriving before or at period $t$

$n_{t} \quad$ Number of elements of the set $\mathcal{M}_{t}$, i.e. $n_{t}=\operatorname{card}\left(\mathcal{M}_{t}\right)$

$p_{b} \quad$ Index of the first period with a possible non-null backlog quantity, $p_{b}=p_{m}$

$p_{B} \quad$ Index of the last period with a possible non-null backlog quantity:

$$
p_{B}=\max _{t=p_{m}, \cdots, p_{M}}\left(t-X_{t}+L_{t}^{+}-1\right)
$$

$p_{h} \quad$ Index of the first period with a possible non-null inventory level:

$$
p_{h}=\min _{t=p_{m}, \ldots, p_{M}}\left(t-X_{t}+L_{t}^{-}\right)
$$

$p_{H} \quad$ Index of the last period with a possible non-null inventory level, $p_{H}=p_{M}-1$

$c^{h} \quad$ Unit inventory holding cost per period

$c^{b} \quad$ Unit backlogging cost per period

$S_{0} \quad$ Safety stock, it is a decision variable and is held when there is a possible shortage

\section{Functions}

$E \llbracket . \rrbracket \quad$ Expected value

$F_{t}($.$) Cumulative distribution function of L_{t}$

$q($.$) Recursive function$

$Q($.$) Recursive function$

$x^{+} \quad$ Maximum value of $x$ and 0 , i.e. $x^{+}=\max (x, 0)$

$x^{-} \quad$ Minimum value of $x$ and 0, i.e. $x^{-}=\min (x, 0)$

$\mathbb{1}_{\{A\}} \quad$ Indicator function, equals 1 if event $A$ is true; 0 otherwise

\subsection{Mathematical model}

The objective is to determine a sequence of planned lead times $\vec{X}$ and safety stock level $S_{0}$, while satisfying all demands and minimizing the total expected cost over the given planning horizon.

We assume that each real lead time $L_{t}$ is a discrete random variable varying between $L_{t}^{-}$and $L_{t}^{+}$and each planned lead time $X_{t} \in\left\{L_{t}^{-}, \ldots, L_{t}^{+}\right\}$. Thus, the total cost is a random variable and its expression is given in Eq. (1): 


$$
\operatorname{TC}\left(S_{0}, \vec{X}, \vec{L}\right)=c^{h} \times \sum_{t=p_{h}}^{p_{H}} S_{t}+c^{b} \sum_{t=p_{b}}^{p_{B}} R_{t}
$$

To evaluate the expected value of the total cost $(E \llbracket T C \rrbracket)$, it is important to determine the expressions of $R_{t}, S_{t}$ and then their respective expected values.

\section{Property 1.}

An explicit form for the backlogging value at period $t$ is the following:

$$
R_{t}=\left(\sum_{\substack{i \in \mathcal{M}_{t} \\ i \leq t}} D_{i}-S_{0}-\sum_{i \in \mathcal{M}_{t}} D_{i} \mathbb{1}_{\left\{i-X_{i}+L_{i} \leq t\right\}}\right)^{+}
$$

\section{Proof.}

The backlogging value at period $t$ is the following:

$$
R_{t}=\left(\sum_{i=p_{m}}^{t} D_{i}-S_{0}-\sum_{i=p_{h}}^{t} A_{i}\right)^{+}
$$

On one hand, the sum of release order quantities at period $t$ contains the set of demands ordered that can arrive before or at period $\left(\mathbb{1}_{\left\{i-X_{i}+L_{i} \leq t\right\}}\right)$ :

$$
\sum_{i=p_{h}}^{t} A_{i}=\sum_{i=p_{m}}^{p_{M}} D_{i} \mathbb{1}_{\left\{i-X_{i}+L_{i} \leq t\right\}}
$$

Knowing that for each demand $D_{i}, L_{i}^{-} \leq L_{i}$. Thus, the sum can be split into two sub-sums as follows:

$$
\sum_{i=p_{h}}^{t} A_{i}=\sum_{i=p_{m}}^{P_{M}} D_{i} \mathbb{1}_{\left\{\begin{array}{l}
i-X_{i}+L_{i}^{+} \leq t \\
i-X_{i}+L_{i}^{-} \leq t
\end{array}\right\}}+\sum_{i=p_{m}}^{p_{M}} D_{i} \mathbb{1}_{\left\{\begin{array}{l}
i-X_{i}+L_{i}^{+}>t \\
i-X_{i}+L_{i}^{-} \leq t
\end{array}\right\}}
$$

The first part contains the demands that arrive at least at period $t\left(i-X_{i}+L_{i}^{+} \leq t\right)$ and the second one contains those that can arrive after $t\left(i-X_{i}+L_{i} \leq t\right.$ and $\left.i-X_{i}+L_{i}^{+}>t\right)$.

Knowing that $\mathcal{M}_{t}$ contains all indexes of periods such that $L_{j}^{-} \leq t-j+X_{j} \leq L_{j}^{+}-1$, then:

$$
\sum_{i=p_{h}}^{t} A_{i}=\sum_{i=0}^{P_{M}} D_{i} \mathbb{1}_{\left\{\begin{array}{l}
i-X_{i}+L_{i}^{+} \leq t \\
i-X_{i}+L_{i}^{-} \leq t
\end{array}\right.}+\sum_{i \in \mathcal{M}_{t}} D_{i} \mathbb{1}_{\left\{i-X_{i}+L_{i} \leq t\right\}}
$$

On the other hand, the sum of demands until period $t$ is the following:

$$
\sum_{i=p_{m}}^{t} D_{i}=\sum_{i=p_{m}}^{p_{M}} D_{i} \mathbb{1}_{\{i \leq t\}}
$$

Some demands can arrive at least at period $t\left(i-X_{i}+L_{i}^{+} \leq t\right)$ and other ones can be delivered after period $\left(i-X_{i}+L_{i}^{+}>t\right)$, then: 


$$
\begin{aligned}
& \sum_{i=p_{m}}^{t} D_{i}=\sum_{i=p_{m}}^{p_{M}} D_{i} \mathbb{1}_{\left\{\begin{array}{c}
i \leq t \\
i-X_{i}+L_{i}^{+} \leq t
\end{array}\right\}}+\sum_{i=p_{m}}^{p_{M}} D_{i} \mathbb{1}_{\left\{\begin{array}{c}
i \leq t \\
i-X_{i}+L_{i}^{+}>t
\end{array}\right\}} \\
& =\sum_{i=p_{m}}^{p_{M}} D_{i} \mathbb{1}_{\left\{\begin{array}{c}
i \leq t \\
i-X_{i}+L_{i}^{+} \leq t \\
i-X_{i}+L_{i}^{-} \leq t
\end{array}\right\}}+\sum_{i=p_{m}}^{p_{M}} D_{i} \mathbb{1}_{\left\{\begin{array}{c}
i \leq t \\
i-X_{i}+L_{i}^{+} \leq t \\
i-X_{i}+L_{i}^{-}>t
\end{array}\right\}}+\sum_{i=p_{m}}^{p_{M}} D_{i} \mathbb{1}_{\left\{\begin{array}{c}
i \leq t \\
i-X_{i}+L_{i}^{+}>t \\
i-X_{i}+L_{i}^{-} \leq t
\end{array}\right\}}+\sum_{i=p_{m}}^{p_{M}} D_{i} \mathbb{1}_{\left\{\begin{array}{c}
i \leq t \\
i-X_{i}+L_{i}^{+}>t \\
i-X_{i}+L_{i}^{-}>t
\end{array}\right\}}
\end{aligned}
$$

since $X_{i} \in\left\{L_{i}^{-}, \ldots, L_{i}^{+}\right\}$, then:

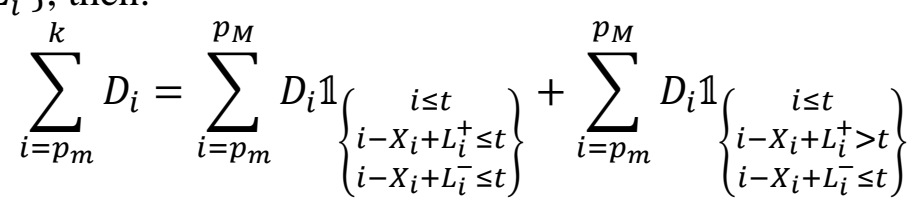

Knowing that $\mathcal{M}_{t}$ contains all indexes of periods such that $L_{j}^{-} \leq t-j+X_{j} \leq L_{j}^{+}-1$, then:

$$
\sum_{i=p_{m}}^{k} D_{i}=\sum_{i=p_{m}}^{p_{M}} D_{i} \mathbb{1}_{\left\{\begin{array}{c}
i \leq t \\
i-X_{i}+L_{i}^{+} \leq t \\
i-X_{i}+L_{i}^{-} \leq t
\end{array}\right\}}+\sum_{\substack{i \in \mathcal{M}_{t} \\
i \leq t}} D_{i}
$$

Using Eq. (4) and Eq. (5), Eq. (2) can be easily deduced.

The backlogging quantity $R_{t}$ at period $t$ is a discrete random variable (because of the randomness of lead times) with a finite number of finite outcomes. The following property gives its expected value.

\section{Property 2.}

An explicit form for the backlogging expected value at period $t$ is the following:

$$
E \llbracket R_{t} \rrbracket=W(t) \times\left(\sum_{\alpha=0}^{n_{k}-1} Q\left(\alpha, \alpha, t, M_{t}\right)\right)
$$

Where the recursive function $Q(., \ldots, .$.$) is defined by:$

$$
Q\left(\alpha, i, t, z_{i+1}\right)=\left\{\begin{array}{cc}
\sum_{\substack{z_{i}=m_{k}+i-1 \\
z_{i} \in \mathcal{M}_{t}}}^{z_{i+1}} Q\left(\alpha, i-1, t, z_{i}\right) & i=0, \alpha=0 \\
\sum_{\substack{z_{i+1} \\
z_{1}=m_{t} \\
z_{1} \in \mathcal{M}_{t}}}\left(U(k)-\sum_{k=1}^{\alpha} D_{z_{k}}\right)^{+} \times V(\alpha, t) & i=1, \forall \alpha
\end{array}\right.
$$

Where

$$
\begin{gathered}
U(t)=\sum_{i \in \mathcal{M}_{t}} D_{i}-S_{0} \\
V(\alpha, t)=\prod_{k=1}^{\alpha}\left(\frac{1}{1-F_{z_{k}}\left(t+X_{z_{k}}-z_{k}\right)}-1\right) \\
W(t)=\prod_{i \in \mathcal{M}_{t}}\left(1-F_{i}\left(t+X_{i}-i\right)\right) \\
F_{i}\left(t+X_{i}-i\right)=P \llbracket L_{i} \leq t+X_{i}-i \rrbracket
\end{gathered}
$$




\section{Proof.}

Let $r_{t}^{\alpha}$ be an element of the subset $\mathcal{R}_{t}^{\alpha}$ of the sample space of the random variable $R_{t}$, where $\alpha \in\left\{0, \ldots, n_{t}-1\right\}$ is the number of order release quantities arriving before or at period $t$. By definition, the expected value of $R_{t}$ is defined as follows:

where

$$
E \llbracket R_{t} \rrbracket=\sum_{\alpha=0}^{n_{t}-1} \sum_{r_{t}^{\alpha} \in \mathcal{R}_{t}^{\alpha}} r_{t}^{\alpha} \times P \llbracket R_{t}=r_{t}^{\alpha} \rrbracket
$$

$$
\mathcal{R}_{t}^{\alpha}=\left\{U(t)-\sum_{k=1}^{\alpha} D_{z_{k}}\right\}_{\substack{z_{\alpha+1}=M_{t} \\ z_{k}=m_{t}+t-1, \ldots, z_{k+1}}}
$$

If no release order quantity arrives before period $t(\alpha=0)$, i.e. all demands indexed by $i \in \mathcal{M}_{t}$ are received after period $t\left(\mathbb{1}_{\left\{L_{i} \geq t+X_{i}-i+1\right\}}=1\right)$, the backlogging value is calculated using Eq. (2) and Eq. (7):

$$
R_{t}=\prod_{i \in \mathcal{M}_{t}} \mathbb{1}_{\left\{L_{i} \geq t+X_{i}-i+1\right\}} \times(U(t))^{+}
$$

In this case, $\mathcal{R}_{t}^{0}$ contains only one element $r_{t}^{0}=(U(t))^{+}$with $P \llbracket R_{t}=r_{t}^{0} \rrbracket=W(t)$, then

$$
P \llbracket R_{t}=r_{t}^{0} \rrbracket \times r_{t}^{0}=W(t) \times(U(t))^{+}
$$

If one release order quantity arrives before period $t(\alpha=1)$, i.e. all demands indexed by $i \in$ $\mathcal{M}_{t}$, except $z_{1}\left(\mathbb{1}_{\left\{L_{z_{1}} \leq t+X_{z_{1}}-z_{1}\right\}}=1\right)$, are received after period $t\left(\mathbb{1}_{\left\{L_{i} \geq t+X_{i}-i+1\right\}}=1\right)$, the backlogging value is calculated using Eq. (2) and Eq. (7):

$$
R_{t}=\left(U(t)-D_{z_{1}} \mathbb{1}_{\left\{L_{z_{1}} \leq t+X_{z_{1}}-z_{1}\right\}}-\sum_{\substack{i \in \mathcal{M}_{t} \\ i \neq z_{1}}} D_{i}\left(1-\mathbb{1}_{\left\{L_{i} \geq t+X_{i}-i+1\right\}}\right)\right)^{+}
$$

Using the fact that $\forall i=p_{m}, \ldots, p_{M}$, the random variables $L_{i}$ are independent, so:

$$
\begin{aligned}
R_{t}=\mathbb{1}_{\left\{L_{z_{1}} \leq t+X_{z_{1}}-z_{1}\right\}} \times\left(U(t)-D_{z_{1}}\right)^{+} \times \prod_{\substack{i \in \mathcal{M} t \\
i \neq z_{1}}} \mathbb{1}_{\left\{L_{i} \geq t+X_{i}-i+1\right\}} \\
=\prod_{i \in \mathcal{M} t_{t}} \mathbb{1}_{\left\{L_{i} \geq t+X_{i}-i+1\right\}} \times\left(U(t)-D_{z_{1}}\right)^{+} \times \frac{\mathbb{1}_{\left\{L_{z_{1}} \leq t+X_{z_{1}}-z_{1}\right\}}}{\mathbb{1}_{\left\{L_{z_{1}} \geq t+X_{z_{1}}-z_{1}+1\right\}}}
\end{aligned}
$$

Note that there is $n_{t}=\operatorname{card}\left(\mathcal{M}_{t}\right)$ possibilities for $z_{1}\left(z_{1}=m_{t}, \ldots, M_{t}\right.$ such as $\left.z_{1} \in \mathcal{M}_{t}\right)$, then:

$$
\sum_{r_{t}^{1} \in \mathcal{R}_{t}^{1}} r_{t}^{1} \times P \llbracket R_{t}=r_{t}^{1} \rrbracket=W(t) \times \sum_{\substack{z_{1}=m_{t} \\ z_{1} \in \mathcal{M}_{t}}}^{M_{t}}\left(\left(U(t)-D_{z_{1}}\right)^{+} \times V(1, t)\right)
$$

If two release order quantities arrive before period $t(\alpha=2)$, i.e. all demands indexed by $i \in$ $\mathcal{M}_{t}$, except two of them $\left(z_{1}, z_{2} \in \mathcal{M}_{t}\right)$, are received after period $t\left(\mathbb{1}_{\left\{L_{z_{1}} \leq t+X_{z_{1}}-z_{1}\right\}} \times\right.$ $\mathbb{1}_{\left\{L_{z_{2}} \leq t+X_{z_{2}}-z_{2}\right\}}=1$ and $\left.\mathbb{1}_{\left\{L_{i} \geq t+X_{i}-i+1\right\}}=1\right)$, the backlogging value is calculated using Eq. (2) and Eq. (7): 


$$
R_{t}=\left(U(t)-\sum_{k=1}^{2} D_{z_{k}} \mathbb{1}_{\left\{L_{z_{k}} \leq t+X_{z_{k}}-z_{k}\right\}}-\sum_{\substack{i \in \mathcal{M}_{t} \\ i \neq\left\{z_{1}, z_{2}\right\}}} D_{i}\left(1-\mathbb{1}_{\left\{L_{i} \geq t+X_{i}-i+1\right\}}\right)\right)^{+}
$$

Using the fact that $\forall i=p_{m}, \ldots, p_{M}$, the random variables $L_{i}$ are independent, so:

$$
\begin{aligned}
R_{t}=\prod_{k=1}^{2}\left(\mathbb{1}_{\left\{L_{z_{k}} \leq t+X_{z_{k}}-z_{k}\right\}}\right) \times\left(U(t)-\sum_{k=1}^{2} D_{z_{k}}\right)^{+} \times \prod_{\substack{i \in \mathcal{M}_{t} \\
i \neq\left\{z_{1}, z_{2}\right\}}} \mathbb{1}_{\left\{L_{i} \geq t+X_{i}-i+1\right\}} \\
=\prod_{i \in \mathcal{M} \mathcal{M}_{t}} \mathbb{1}_{\left\{L_{i} \geq t+X_{i}-i+1\right\}} \times\left(U(t)-\sum_{k=1}^{2} D_{z_{k}}\right)^{+} \times \prod_{k=1}^{2}\left(\frac{\mathbb{1}_{\left\{L_{z_{k}} \leq t+X_{z_{k}}-z_{k}\right\}}}{\left.\mathbb{1}_{\left\{L_{z_{k}} \geq t+X_{z_{k}}-z_{k}+1\right\}}\right)}\right)
\end{aligned}
$$

Note that there is $\left(\begin{array}{c}n_{t} \\ 2\end{array}\right)$ possibilities for $\left(z_{1}, z_{2}\right)$ such as $z_{2}=m_{t}+1, \ldots, M_{t}, z_{1}=m_{t}, \ldots, z_{2}$ and $z_{1}, z_{2} \in \mathcal{M}_{t}$, then:

$$
\sum_{r_{t}^{2} \in \mathcal{R}_{t}^{2}} r_{t}^{2} \times P \llbracket R_{t}=r_{t}^{2} \rrbracket=W(t) \times \sum_{\substack{z_{2}=m_{t}+1 \\ z_{2} \in \mathcal{M}_{t}}}^{M_{t}} \sum_{\substack{z_{1}=m_{t} \\ z_{1} \in \mathcal{M}_{t}}}^{z_{2}}\left(\left(U(t)-\sum_{k=1}^{2} D_{z_{k}}\right)^{+} \times V(2, t)\right)
$$

The general formulation of $E \llbracket R_{t} \rrbracket$ can easily be expressed recursively by using Eq. (8), Eq. (9) and Eq. (10)

The inventory level $S_{t}$ at period $t$ is also a discrete random variable with a finite number of finite outcomes. The following property gives its expected value.

\section{Property 3.}

An explicit form for expected value of the inventory level $S_{t}$ at period $t$ is given by the following equation:

$$
E \llbracket S_{t} \rrbracket=S_{0}+\sum_{i=p_{h}}^{t} E \llbracket A_{i} \rrbracket-\sum_{i=p_{m}}^{t} D_{i}+E \llbracket R_{t} \rrbracket
$$

where

$$
E \llbracket A_{i} \rrbracket=\sum_{j \in \mathcal{A}_{i}} D_{j} \times P \llbracket L_{j}=i-j+X_{j} \rrbracket
$$

\section{Proof.}

The inventory level $S_{t}$ at period $t$ is the following:

$$
S_{t}=\left(S_{0}+\sum_{i=p_{h}}^{t} A_{i}-\sum_{i=p_{m}}^{t} D_{i}\right)^{+}
$$

Using the property $x=x^{+}+x^{-}$and Eq. (3), we obtain:

$$
S_{t}=R_{t}+S_{0}+\sum_{i=p_{h}}^{t} A_{i}-\sum_{i=p_{m}}^{t} D_{i}
$$


and then $E \llbracket S_{t} \rrbracket$ can be deduced.

The demand being deterministic and dynamic between periods $p_{m}$ and $p_{M}$, the backlogging quantity $R_{t}$ can be positive only for periods between $p_{b}=p_{m}$ and $p_{B}=\max _{t=p_{m}, \cdots, p_{M}}\left(t-X_{t}+\right.$ $\left.L_{t}^{+}-1\right)$ and the inventory level $S_{t}$ can be positive only for periods between $p_{h}$ and $p_{H}$. The expected value of the total cost can be expressed at the following property.

\section{Property 4.}

$$
\begin{aligned}
& E \llbracket T C \rrbracket=c^{h}\left(p_{H}-p_{h}+1\right) S_{0}+\left(c^{h}+c^{b}\right) \sum_{t=p_{b}}^{p_{H}} E \llbracket R_{t} \rrbracket+c^{b} \sum_{t=p_{M}}^{p_{B}} \times E \llbracket R_{t} \rrbracket \\
& \quad+c^{h}\left(\sum_{t=0}^{p_{H}-p_{h}}\left(p_{H}-p_{h}+1-t\right) \times E \llbracket A_{p_{h}+t} \rrbracket-\sum_{t=0}^{p_{H}-p_{m}}\left(p_{H}-p_{m}+1-t\right) \times D_{p_{m}+t}\right)
\end{aligned}
$$

\section{Proof.}

Using Eq. (1), the explicit form of the expected value of the total cost can be deduced as follows:

Using Eq. (11), we obtain:

$$
E \llbracket T C \rrbracket=\sum_{t=p_{h}}^{p_{H}} c^{h} \times E \llbracket S_{t} \rrbracket+\sum_{t=p_{b}}^{p_{B}} c^{b} \times E \llbracket R_{t} \rrbracket
$$

$$
E \llbracket T C \rrbracket=c^{h} S_{0} \sum_{t=p_{h}}^{p_{H}} 1+c^{h} \sum_{t=p_{h}}^{p_{H}}\left(\sum_{i=p_{h}}^{t} E \llbracket A_{i} \rrbracket-\sum_{i=p_{m}}^{t} D_{i}\right)+c^{h} \sum_{t=p_{h}}^{p_{H}} E \llbracket R_{t} \rrbracket+c^{b} \sum_{t=p_{b}}^{p_{B}} E \llbracket R_{t} \rrbracket
$$

Knowing that

$$
\begin{aligned}
& \sum_{t=p_{b}}^{p_{B}} E \llbracket R_{t} \rrbracket=\sum_{t=p_{b}}^{p_{H}} E \llbracket R_{t} \rrbracket+\sum_{t=p_{M}}^{p_{B}} \times E \llbracket R_{t} \rrbracket \\
& \sum_{t=p_{h}}^{p_{H}} E \llbracket R_{t} \rrbracket=\sum_{t=p_{b}}^{p_{H}} E \llbracket R_{t} \rrbracket \\
& \sum_{t=p_{h}}^{p_{H}} \sum_{i=p_{h}}^{t} E \llbracket A_{i} \rrbracket=\sum_{t=0}^{p_{H}-p_{h}}\left(p_{H}-p_{h}+1-t\right) \times E \llbracket A_{p_{h}+t} \rrbracket \\
& \sum_{t=p_{h}}^{p_{H}} \sum_{i=p_{m}}^{t} D_{i}=\sum_{t=0}^{p_{H}-p_{m}}\left(p_{H}-p_{m}+1-t\right) \times D_{p_{m}+t}
\end{aligned}
$$

Then, the explicit form of the expected value of the total cost can be deduced.

\section{Resolving approaches}

To determine planned lead times and safety stock level which minimize the total expected cost, four approaches are applied:

- NB\&SS: it is based on Newsboy formula presented in (Ben Ammar et al. 2017) and an iterative method. The sequence of optimal planned lead times is given by the Newsboy formula and then the optimal safety stock is determined iteratively. 
- Xmin\&SS: it consists of ordering all quantities as late as possible, the sequence of planned lead times is composed of minimal values of real times $\left(X_{t}=L_{t}^{-} \forall t\right)$. Then, the optimal safety stock level is also determined iteratively.

- Xmax: it consists of ordering all quantities as soon as possible, i.e. the sequence of planned lead times is composed of maximal values of real times $\left(X_{t}=L_{t}^{+} \forall t\right)$.

- Xopt\&SS: it employs a classical GA to determine the sequence of optimal planned lead times for a given safety stock level. The GA is run for several values of safety stock and then the best solution is selected.

Note that for the GA, an "Elitist Strategy" is employed. The initial population is randomly generated. Each individual is a sequence of planned lead times of the demands. Each gene contains a planned lead time $X_{t}$ which may take any value between $L^{-}$and $L^{+}$. The expected value of the total cost $E \llbracket T C \rrbracket$ is the fitness function used to evaluate each solution.

\section{Experiments and discussions}

The resolving approaches, mentioned above, have been tested on 12 data set combinations. The characteristics of data sets and the different combinations are given in Table 1 and Table 2. Each combination has been tested through the resolution of 100 instances where the vector of demand is generated randomly. For all instances, the demand is generated for periods between $p_{m}=11$ and $p_{M}=25$. Table 3 gives the probability distributions of demands' lead times.

\begin{tabular}{|c|c|c|c|c|c|c|c|}
\hline \multirow{2}{*}{$\begin{array}{c}\text { Inventory } \\
\text { cost }\end{array}$} & \multicolumn{2}{|c|}{ Backlogging cost } & \multicolumn{4}{c|}{ Demand variability } \\
\cline { 2 - 8 } & Low & Med. & High & Zero & Low & Med. & High \\
\hline 6 & 7 & 15 & 25 & $D=100$ & $D \sim U(90,110)$ & $D \sim U(70,130)$ & $D \sim U(0,200)$ \\
\hline
\end{tabular}

Table 1. Characteristics of data sets.

\begin{tabular}{|l|c|c|c|c|c|c|c|c|c|c|c|c|}
\hline Data set combination & 1 & 2 & 3 & 4 & 5 & 6 & 7 & 8 & 9 & 10 & 11 & 12 \\
\hline Demand variability & $\mathrm{Z}$ & $\mathrm{Z}$ & $\mathrm{Z}$ & $\mathrm{L}$ & $\mathrm{L}$ & $\mathrm{L}$ & $\mathrm{M}$ & $\mathrm{M}$ & $\mathrm{M}$ & $\mathrm{H}$ & $\mathrm{H}$ & $\mathrm{H}$ \\
\hline Backlogging cost $\left(c^{b}\right)$ & $\mathrm{L}$ & $\mathrm{M}$ & $\mathrm{H}$ & $\mathrm{L}$ & $\mathrm{M}$ & $\mathrm{H}$ & $\mathrm{L}$ & $\mathrm{M}$ & $\mathrm{H}$ & $\mathrm{L}$ & $\mathrm{M}$ & $\mathrm{H}$ \\
\hline
\end{tabular}

Table 2. Data set combinations. $Z=Z e r o, M=$ Medium, $L=L o w, H=H i g h$.

\begin{tabular}{|c|c|c|c|c|c|c|c|}
\hline $\boldsymbol{l}$ & $\mathbf{1}$ & $\mathbf{2}$ & $\mathbf{3}$ & $\mathbf{4}$ & $\mathbf{5}$ & $\mathbf{6}$ & $\mathbf{7}$ \\
\hline $\operatorname{Pr} \llbracket \boldsymbol{L}_{\mathbf{1 1}}=\boldsymbol{l} \rrbracket$ & 0.27 & 0.51 & 0.2 & 0.01 & 0.01 & 0 & 0 \\
\hline $\operatorname{Pr} \llbracket \boldsymbol{L}_{\mathbf{1 2}}=\boldsymbol{l} \rrbracket$ & 0 & 0.2 & 0.3 & 0.25 & 0.25 & 0 & 0 \\
\hline $\operatorname{Pr} \llbracket \boldsymbol{L}_{\mathbf{1 3}}=\boldsymbol{l} \rrbracket$ & 0 & 0 & 0.2 & 0.2 & 0.2 & 0.2 & 0.2 \\
\hline $\operatorname{Pr} \llbracket \boldsymbol{L}_{\mathbf{1 4}}=\boldsymbol{l} \rrbracket$ & 0 & 0.25 & 0.3 & 0.25 & 0.2 & 0 & 0 \\
\hline $\operatorname{Pr} \llbracket \boldsymbol{L}_{\mathbf{1 5}}=\boldsymbol{l} \rrbracket$ & 0 & 0 & 0.5 & 0.5 & 0 & 0 & 0 \\
\hline $\operatorname{Pr} \llbracket \boldsymbol{L}_{\mathbf{1 6}}=\boldsymbol{l} \rrbracket$ & 0 & 0.3 & 0.3 & 0.4 & 0 & 0 & 0 \\
\hline $\operatorname{Pr} \llbracket \boldsymbol{L}_{\mathbf{1 7}}=\boldsymbol{l} \rrbracket$ & 0 & 0 & 0.5 & 0.5 & 0 & 0 & 0 \\
\hline $\operatorname{Pr} \llbracket \boldsymbol{L}_{\mathbf{1 8}}=\boldsymbol{l} \rrbracket$ & 0 & 0.2 & 0.8 & 0 & 0 & 0 & 0 \\
\hline $\operatorname{Pr} \llbracket \boldsymbol{L}_{\mathbf{1 9}}=\boldsymbol{l} \rrbracket$ & 0 & 0.6 & 0.4 & 0 & 0 & 0 & 0 \\
\hline $\operatorname{Pr} \llbracket \boldsymbol{L}_{\mathbf{2 0}}=\boldsymbol{l} \rrbracket$ & 0.2 & 0.2 & 0.2 & 0.2 & 0.2 & 0 & 0 \\
\hline $\operatorname{Pr} \llbracket \boldsymbol{L}_{\mathbf{2 1}}=\boldsymbol{l} \rrbracket$ & 0 & 0.25 & 0.25 & 0.25 & 0.25 & 0 & 0 \\
\hline $\operatorname{Pr} \llbracket \boldsymbol{L}_{\mathbf{2 2}}=\boldsymbol{l} \rrbracket$ & 0 & 0 & 0.3 & 0.2 & 0.2 & 0.2 & 0.1 \\
\hline $\operatorname{Pr} \llbracket \boldsymbol{L}_{\mathbf{2 3}}=\boldsymbol{l} \rrbracket$ & 0 & 0.25 & 0.25 & 0.25 & 0.25 & 0 & 0 \\
\hline $\operatorname{Pr} \llbracket \boldsymbol{L}_{\mathbf{2 4}}=\boldsymbol{l} \rrbracket$ & 0 & 0 & 0.5 & 0.5 & 0 & 0 & 0 \\
\hline $\operatorname{Pr} \llbracket \boldsymbol{L}_{\mathbf{2 5}}=\boldsymbol{l} \rrbracket$ & 0 & 0.3 & 0.3 & 0.4 & 0 & 0 & 0 \\
\hline
\end{tabular}

Table 3. Probability distributions of lead times 
Figure 2 shows the variation of the expected total cost for data combination 8 as function of safety stock level. The best known solution is the one found by Xopt\&SS. It is equal to 6284.5. The best solution found by $N B \& S S$ corresponds to an expected total cost of 7164.9 and the best one found by Xmax is equal to 11867.1 . For these three approaches, introducing the safety stock seems to be useless regarding costs. For Xmin\&SS, the optimal solution (10601) corresponds to a non-null average safety stock $\left(S_{0}=124.6\right)$.

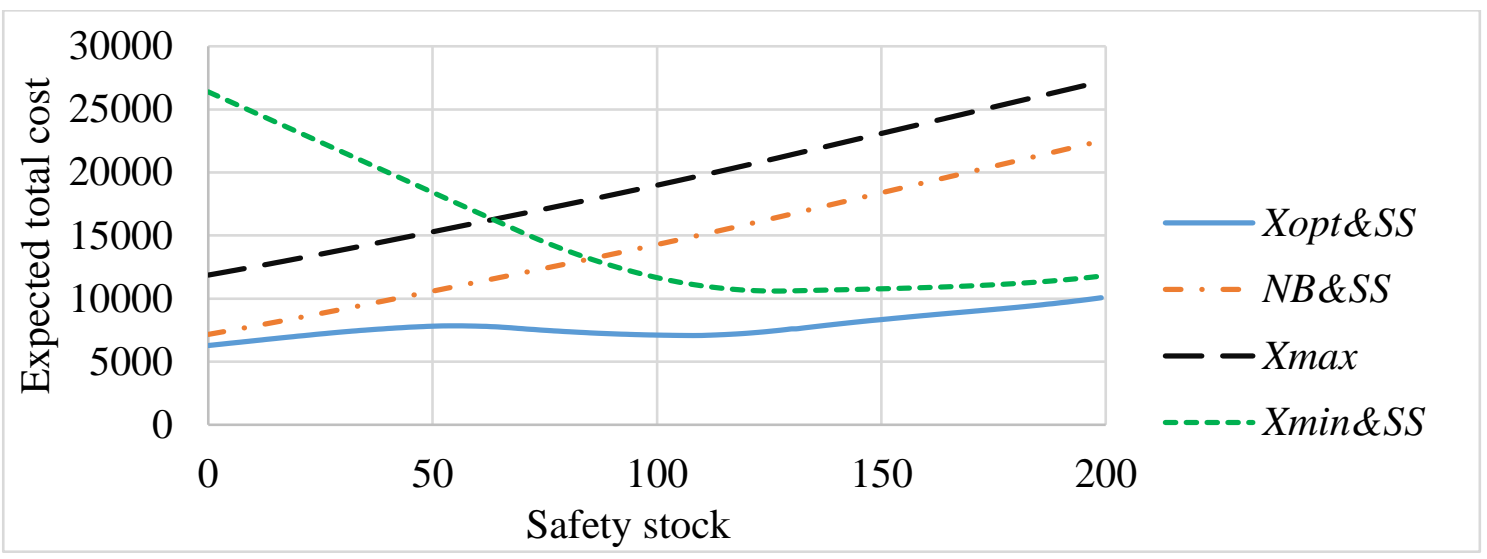

Figure 2. Variation of the expected total cost as function of safety stock level.

Table 4 shows the average results of the 4 approaches for the 12 data set combinations. It can be seen that, whatever are the demand variability and backlogging cost, the Xopt\&SS approach dominates all other approaches. For Xmin \&SS approach, safety stock level it is much more influenced by the increase of backlogging cost. For a high backlogging cost, the increase of demand variability decreases slightly the level of safety stock.

The relative performances of the different approaches for all data set combinations are summarized in Figure 3, where the Xopt\&SS approach is taken as reference. Globally, both $N B \& S S$ and Xopt\&SS approaches dominate the two others (Xmax and Xmin\&SS). The average gap between the first two ones decreases as function of demand variability. Nevertheless, it is small only if the backlogging cost is low and still relatively great otherwise. Concerning Xmax approach, even if it seems to be greatly inefficient, especially for the case of low backlogging cost, it becomes more efficient than Xmin \&SS when the backlogging cost is high or when this cost is medium and the demand variability is high.

\begin{tabular}{|c|c|c|c|c|c|c|c|c|}
\hline \multirow{2}{*}{$\begin{array}{c}\text { Data set } \\
\text { combination }\end{array}$} & \multicolumn{2}{|c|}{ Xopt\&SS } & \multicolumn{2}{c|}{ NB\&SS } & \multicolumn{2}{c|}{ Xmax } & \multicolumn{2}{c|}{ Xmin\&SS } \\
\cline { 2 - 9 } & $S_{0}$ & ETC & $S_{0}$ & ETC & $S_{0}$ & ETC & $S_{0}$ & ETC \\
\hline 1 & 0 & 4287.6 & 0 & 4820.3 & 0 & 11742.0 & 100 & 6266.6 \\
\hline 2 & 0 & 5861.6 & 0 & 6916.1 & 0 & 11742.0 & 100 & 10208.8 \\
\hline 3 & 0 & 6995.3 & 0 & 10026.9 & 0 & 11742.0 & 200 & 11679.7 \\
\hline 4 & 0 & 4389.6 & 0 & 4852.5 & 0 & 11638.6 & 100.6 & 6441.4 \\
\hline 5 & 0 & 5987.8 & 0 & 6935.0 & 0 & 11658.7 & 107.8 & 10204.7 \\
\hline 6 & 0 & 7094.5 & 0 & 9976.7 & 0 & 11673.3 & 195.1 & 11899.0 \\
\hline 7 & 0 & 4599.2 & 0 & 5023.4 & 0 & 11810.1 & 104.5 & 6924.9 \\
\hline 8 & 0 & 6284.5 & 0 & 7164.9 & 0 & 11867.1 & 124.6 & 10601.0 \\
\hline 9 & 0 & 7359.3 & 0 & 10005.0 & 0 & 11651.4 & 187.3 & 12512.4 \\
\hline 10 & 0 & 5136.5 & 0 & 5467.2 & 0 & 11823.3 & 104.2 & 8548.2 \\
\hline 11 & 0 & 6805.3 & 0 & 7657.6 & 0 & 11164.1 & 157.7 & 12364.8 \\
\hline 12 & 0 & 8002.1 & 0 & 9989.3 & 0 & 11612.7 & 184.5 & 15063.2 \\
\hline
\end{tabular}

Table 4. Average results for all data set combinations 


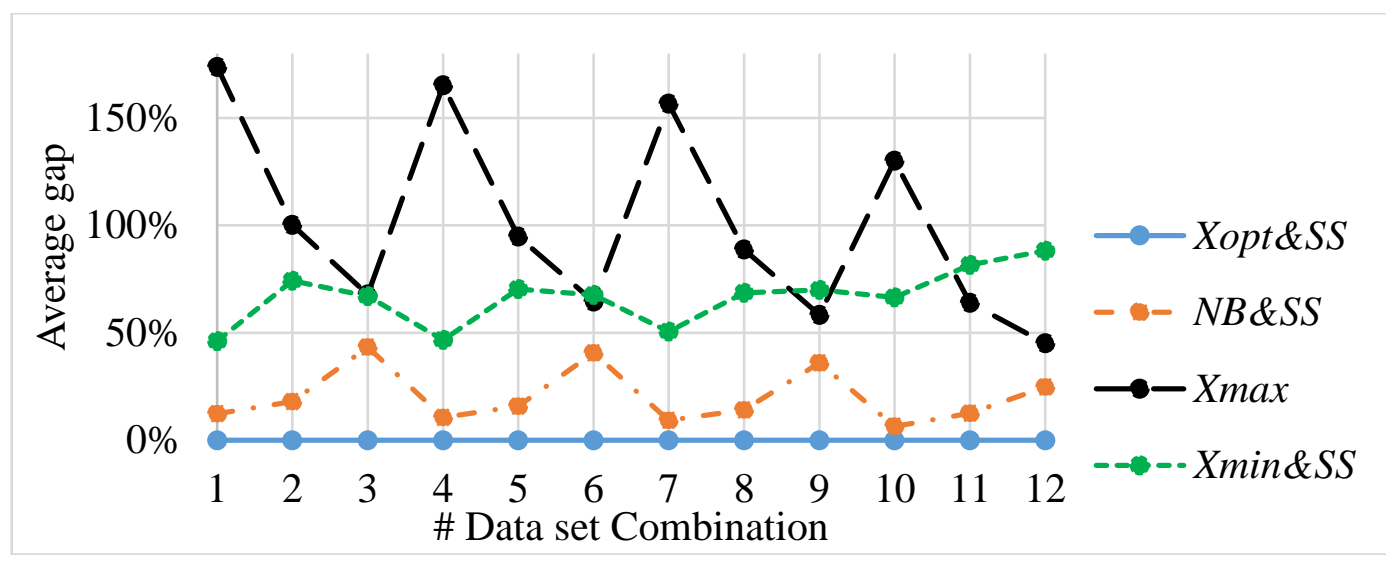

Figure 3. Relative performances of the different approaches

To analyze the effect of lead times variability on the robustness of the Xopt\&SS approach, the effect of variance is studied. To do so, we proceed by variating the variance of demands' lead times. For all periods, we assume the same probability distributions: $\operatorname{Pr} \llbracket L_{t}=1 \rrbracket=0.245$, $\operatorname{Pr} \llbracket L_{t}=2 \rrbracket=0.48, \operatorname{Pr} \llbracket L_{t}=3 \rrbracket=0.255, \operatorname{Pr} \llbracket L_{t}=4 \rrbracket=0.01$ and $\operatorname{Pr} \llbracket L_{t}=5 \rrbracket=0.01$.

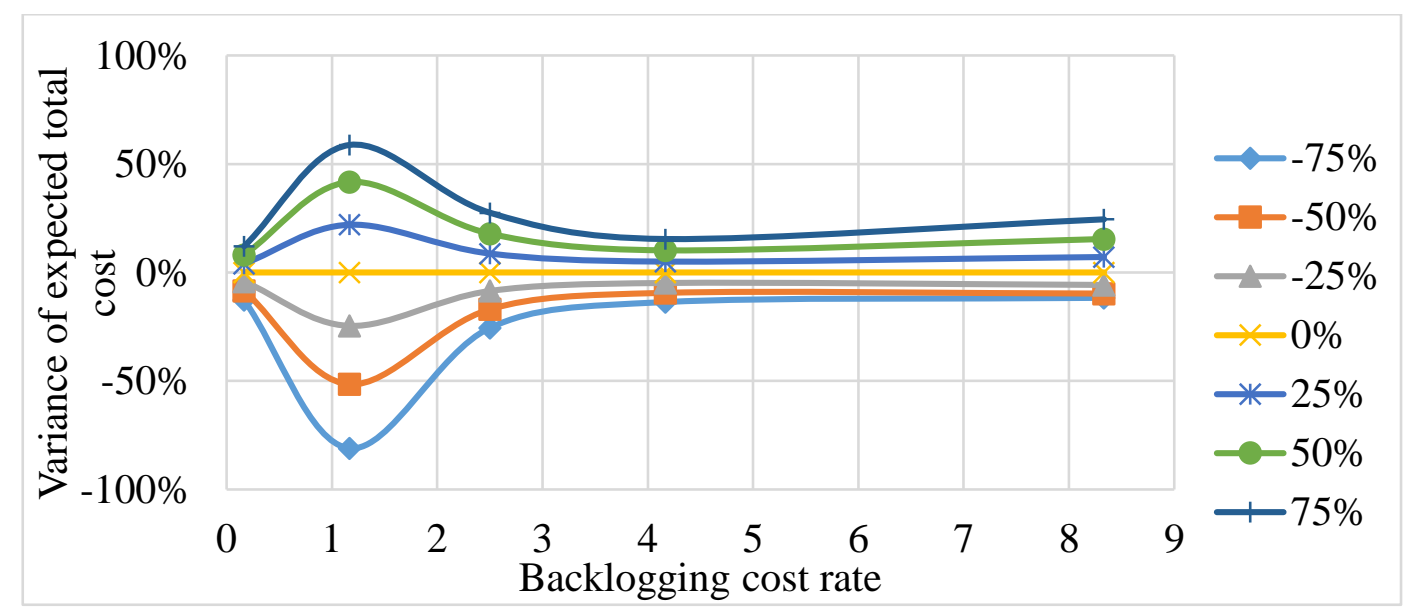

Figure 4. Effect of lead time variance

In Figure 4, we analyse the effect of variance. It varies between $-75 \%$ and $75 \%$ compared to the reference case. We notice that the variation of the expected total cost increases when the ratio $c^{b} / c^{h}$ is close to 1 whatever the variability of the variance of the lead time is. However, the variance of expected total cost depends on the estimation of the probability distribution of the lead times. When the variance of lead times increases the variance of the expected total cost is higher. It means that, it should be better to overestimate the variability of the lead times.

It is worthwhile to mention that in the case of backlogging cost close to the holding cost, it seems to be important to obtain reliable statistical data to get a good estimate of the probability distributions of lead times and/or push the suppliers to be more responsive able to cope with the sources of the uncertainty regarding their lead times.

\section{Conclusions}

This paper deals with a multi-period production planning with a known dynamic demand and stochastic lead times. A general probabilistic model, including a recursive procedure to calculate the expected total cost as function of planned lead times and safety stock level, is proposed and solved using a classical genetic algorithm. This approach is compared to three 
other ones to illustrate its performance. The results prove that, under certain assumptions, it could be advantageous to optimizing planned lead times rather than implementing safety stocks. A sensitivity analysis is also used to understand the effect of lead time dispersion on the quality of the solution.

Future work will focus on improving the genetic algorithm performances and testing this approach on industrial cases. The used modelling mechanism will enable us to extend the model to multi-level assembly systems.

\section{References}

Axsäter S., 2005. Planning order releases for an assembly system with random operation times. Operations Research Spectrum, 27, 459-470.

Ben-Ammar, O., Bettayeb, B., Dolgui, A. 2017. Supply planning optimization for linear production system with stochastic lead-times and quality control. In International Conference on Industrial Engineering and Systems Management (IESM 2017).

Ben-Ammar, O., Dolgui, A., Wu, D.D., 2017. Planned lead times optimization for multi-level assembly systems under uncertainties. Omega, https://doi.org/10.1016/j.omega.2017.11.004.

Chopra, S., Reinhardt, G., Dada, M., 2004. The effect of lead time uncertainty on safety stocks. Decision Sciences, 35(1), 1-24.

Chu, C., Proth, J.-M., Xie, X., 1993. Supply management in assembly systems. Naval Research Logistics, 40(7), 933-949.

Deng, J., Song, P.J.S., Zhang, B., 2010. An inventory model with stochastic lead-time and stochastic demand. In Control and Automation (ICCA), 8th IEEE International Conference on Control and Automation, 1778-1782.

Dolgui, A., Ben-Ammar, O., Hnaien, F., Louly, M.A., 2013. A state of the art on supply planning and inventory control under lead time uncertainty. Studies in Informatics and Control, 22(3), 255-268.

He, X. J., Kim, J. G., Hayya, J. C., 2005. The cost of lead-time variability: The case of the exponential distribution. International Journal of Production Economics, 97(2), 130-142.

Jansen, M.M., de Kok, T.G., Fransoo, J.C., 2013. Lead time anticipation in Supply Chain operations planning, OR Spectrum, 35(1), 251-290.

Minner, S., 2003. Multiple-supplier inventory models in supply chain management: A review. International Journal of Production Economics, 81, 265-279.

Muthuraman, K., Seshadri, S., Wu, Q., 2014. Inventory management with stochastic lead times. Mathematics of Operations Research, 40(2), 302-327.

Ould Louly, M.-A., Dolgui, A., 2013. Optimal MRP parameters for a single item inventory with random replenishment lead time, POQ policy and service level constraint. International Journal of Production Economics, 143(1), 35-40.

Porteus, E. L., 2002. Foundations of stochastic inventory theory. Stanford University Press.

Rossi, R., Tarim, S.A., Bollapragada, R., 2012. Constraint-based local search for inventory control under stochastic demand and lead time. INFORMS Journal on Computing, 24 (1), 66-80.

Tang, O., Grubbström, R.W., 2003. The detailed coordination problem in a two-level assembly system with stochastic lead times. International Journal of Production Economics, 81-82, 415-429.

Van Kampen, T.J., Van Donk, D.P., Van Der Zee, D.J., 2010. Safety stock or safety lead time: coping with unreliability in demand and supply. International Journal of Production Research, 48(24), 7463-7481.

Wang, P., Hill, J. A., 2006. Recursive Behavior of Safety Stock Reduction: The Effect of LeadTime Uncertainty. Decision Sciences, 37(2), 285-290.

Yano C.A., 1987. Stochastic Leadtimes in Two-Level Assembly Systems. IIE Transactions, 19, 371-378. 